\title{
Selpercatinib Is a Promising Treatment Option for RET-Mutated Medullary and RET Fusion-Positive Thyroid Cancers
}

\section{Tyler Drake}

Division of Endocrinology and Metabolism; Department of Medicine; Minneapolis VA Healthcare System; University of Minnesota Medical School; Minneapolis, Minnesota, U.S.A.

Review of: Wirth LJ, Sherman E, Robinson B, Solomon B, Kang H, Lorch J, Worden F, Brose M, Patel J, Leboulleux S, et al. 2020 Efficacy of selpercatinib in RET-altered thyroid cancers. N Engl J Med 383:825835. PMID: 32846061.

\section{SUMMARY}

\section{Background}

An uncommon type of thyroid cancer is medullary thyroid carcinoma (MTC), which accounts for roughly $5 \%$ of all thyroid cancers (1). RET mutations are common in MTC and can be due either to germline mutations such as multiple endocrine neoplasia type 2 (accounting for $\sim 25 \%$ of MTCs) or somatic mutations (seen in $\sim 60 \%$ of sporadic MTCs), while RET fusions can be present but are rare in differentiated and anaplastic thyroid cancers, accounting for $<10 \%$ of all such cancers $(2,3)$.

In patients with MTC, RET mutations are associated with a more aggressive course. Treatment options for MTC include surgery, external-beam radiation, and multitargeted kinase inhibitors such as vandetanib and cabozantinib $(4,5)$, with the latter option of systemic therapy generally reserved for progressive or metastatic disease. Unfortunately, the use of vandetanib and cabozantinib is often limited by their toxic side effects, often requiring either a dose reduction or treatment discontinuation in many patients. This study (6) examined the efficacy and safety of selpercatinib, a highly selective small-molecule RET inhibitor, in patients with RET-mutated MTC and RET fusion-positive thyroid cancer.

\section{Methods}

This was an open-label, multisite phase 1-2 clinical trial of selpercatinib in patients $\geq 12$ years of age with RET-mutated MTC (with or without previous vandetanib or cabozantinib treatment) and patients with RET fusion-positive thyroid cancer (LIBRETTO-001 trial). All subjects were consecutively enrolled and had an advanced or metastatic solid thyroid cancer tumor meeting the above criteria; tumor progression was not an explicit inclusion criterion, but systemic therapy had to be indicated. Selpercatinib was given continuously in 28-day cycles until disease progression, death, unacceptable toxic effects, or withdrawal from the study. Subjects enrolled in the phase 1 portion of the study received doses ranging from $20 \mathrm{mg}$ once daily to $240 \mathrm{mg}$ twice daily. All subjects enrolled in phase 2 received the recommended dose of $160 \mathrm{mg}$ twice daily.

The primary outcome was an objective response (a complete or partial response) as determined by an independent review committee of expert radiologists. Secondary outcomes included the duration of response, progression-free survival, and safety. Radiologic tumor assessments were done at baseline, every 8 weeks for 1 year, and every 12 

Thyroid Cancers

weeks thereafter. Serum calcitonin and carcinoembryonic antigen (CEA) concentrations were followed in the subjects with MTC.

\section{Results}

A total of 162 subjects were treated with selpercatinib, including 55 with RET-mutated MTC previously treated with vandetanib and/or cabozantinib, 88 with RET-mutated MTC not previously treated with vandetanib and/or cabozantinib, and 19 with RET fusion-positive previously treated thyroid cancer. Of the latter group, 13 had papillary, 3 poorly differentiated, 2 anaplastic, and 1 Hürthle-cell thyroid carcinoma.

Of the 55 subjects with RET-mutated MTC previously treated with vandetanib and/or cabozantinib, $69 \%$ had an objective response $(95 \% \mathrm{Cl}, 55-81 \%)$, with 5 subjects having a complete response and 33 a partial response. Responses were observed regardless of previous treatments and were seen across all qualifying RET mutations. At 1 year, $86 \%$ of the responses were ongoing $(95 \% \mathrm{Cl}, 67-95 \%)$ and $82 \%$ of all patients remained progression-free $(95 \% \mathrm{Cl}$, $69-90 \%)$. A total of $91 \%$ (95\% Cl, 80-97\%) had a biochemical response in their calcitonin levels.

Of the 88 subjects with RET-mutated MTC not previously treated with vandetanib and/or cabozantinib, $73 \%$ had an objective response $(95 \% \mathrm{Cl}, 62-82 \%)$, with 10 subjects having a complete response and
54 a partial response. At 1 year, $91 \%$ of responses $(95 \% \mathrm{Cl}, 72-97 \%)$ were ongoing and $92 \%(95 \% \mathrm{Cl}$, $82-97 \%$ ) of the patients were progression-free.

Of the 19 subjects with RET fusion-positive previously treated thyroid cancer, $79 \%$ had an objective response $(95 \% \mathrm{Cl}, 54-94 \%)$. At 1 year, $71 \%$ of responses were ongoing $(95 \% \mathrm{Cl}, 39-88 \%)$ and $64 \%$ $(95 \% \mathrm{Cl}, 37-82 \%)$ of the patients were progression-free.

The most common grade 3 or 4 adverse events were hypertension (21\% of subjects), increased serum alanine aminotransferase levels (11\%), increased serum aspartate aminotransferase levels ( $9 \%$ ), hyponatremia (8\%), and diarrhea (6\%). Overall, $30 \%$ of subjects required a dose reduction because of treatment-related adverse events, and $2 \%$ discontinued selpercatinib because of treatment-related adverse events.

\section{Conclusions}

In this phase 1-2 clinical trial, selpercatinib (a selective RET inhibitor) showed durable efficacy with mainly low-grade toxicity in patients with RET-mutated MTC with and without previous vandetanib or cabozantinib treatment and in patients with RET fusion-positive previously treated thyroid cancers. Efficacy was seen across all RET alterations and histologic types of thyroid cancers.

\section{COMMENTARY}

This study (6) shows that selpercatinib, a selective RET inhibitor, is a promising and durable treatment option for RET-altered thyroid cancers, most importantly MTC, and has a favorable side-effect profile. These findings resulted in its accelerated approval by the U.S. Food and Drug Administration for the treatment of RET-altered MTCs in May 2020 (7).

Medullary thyroid cancer is far more aggressive than most differentiated thyroid cancers and therefore 

Thyroid Cancers

requires more aggressive treatments. Surgery and external-beam radiation are often used to control locoregional disease in the neck, but when there is progression and/or distant metastasis, systemic therapy is often recommended (4,5). Currently, the multitargeted kinase inhibitors vandetanib and cabozantinib are approved for the treatment of metastatic MTC. However, in my experience, use of these treatments is often limited owing to side effects; in some cases, patients have refused to start these medications out of fear of potential side effects. Selpercatinib offers another systemic treatment option with what appear to be less severe side effects.

In this study, approximately $70 \%$ of patients treated with selpercatinib had an objective response that was consistent across all groups. This compares favorably to the phase 3 trials for cabozantinib (8) and vandetanib (9) which had shown an objective response in $28 \%$ of patients treated with cabozantinib and $45 \%$ with vandetanib. While these trials differed in eligibility criteria, it does provide a helpful comparison. Also, in these phase 3 trials, $12 \%$ of subjects receiving vandetanib and $16 \%$ receiving cabozantinib permanently discontinued the medication because of side effects $(7,8)$, compared to only $2 \%$ receiving selpercatinib (6).

The major limitation of this phase 1-2 trial is that it was an open-label, non-placebo-controlled trial, making it difficult to truly compare it to the current standard of care for advanced MTC. Given these promising results, further study with a phase 3 clinical trial is expected for this treatment option. Effective screening approaches in patients with MTC to identify underlying germline or somatic RET mutations will be essential in the future use of this highly selective RET inhibitor.

\section{References}

1. Sherman SI 2003 Thyroid carcinoma. Lancet 361:501-511.

2. Ciampi R, Romei C, Ramone T, Prete A, Tacito A, Cappagli V, Bottici V, Viola D, Torregrossa L, Ugolini C, et al. 2019 Genetic landscape of somatic mutations in a large cohort of sporadic medullary thyroid carcinomas studied by next-generation targeted sequencing. iScience 20:324-336.

3. Pozdeyev N, Gay LM, Sokol ES, Hartmaier R, Deaver KE, Davis S, French JD, Borre PV, LaBarbera DV, Tan AC, et al. 2018 Genetic analysis of 779 advanced differentiated and anaplastic thyroid cancers. Clin Cancer Res 24:3059-3068.
4. Wells SA, Asa SL, Dralle H, Elisei R, Evans DB, Gagel RF, Lee N, Machens A, Moley JF, Pacini F, et al. 2015 Revised American Thyroid Association guidelines for the management of medullary thyroid carcinoma. Thyroid 25:567-610.

5. Haddad RI, Lydiatt WM, Bischoff L, Busaidy NL, Byrd D, Callender G, Dickson P, Duh Q-Y, Ehya H, Haymart, et al. 2020 NCCN Clinical Practice Guidelines in Oncology: Thyroid carcinoma. Accessed at https://www.nccn.org/professionals/ physician_gls/pdf/thyroid.pdf. 

Thyroid Cancers

6. Wirth LJ, Sherman E, Robinson B, Solomon B, Kang H, Lorch J, Worden F, Brose M, Patel J, Leboulleux $\mathrm{S}$, et al. 2020 Efficacy of selpercatinib in RET-altered thyroid cancers. N Engl J Med 383:825-835.

7. Food and Drug Administration. FDA approves selpercatinib for lung and thyroid cancers with RET gene mutations or fusions. Accessed at https://www. fda.gov/drugs/drug-approvals-and-databases/fdaapproves-selpercatinib-lung-and-thyroid-cancersret-gene-mutations-or-fusions\#: :text=On\%20 May\%208\%2C\%202020\%2C\%20the,cell\%20 lung\%20cancer\%20(NSCLC)\%3B.
8. Elisei R, Schlumberger MJ, Müller SP, Schöffski P, Brose MS, Shah MH, Licitra L, Jarzab B, Medvedev V, KreissI MC, et al. 2013 Cabozantinib in progressive medullary thyroid cancer. J Clin Oncol 31:36393646.

9. Wells SA Jr, Robinson BG, Gagel RF, Dralle H, Fagin JA, Santoro M, Baudin E, Elisei R, Jarzab B, Vasselli JR, et al. 2012 Vandetanib in patients with locally advanced or metastatic medullary thyroid cancer: A randomized, double-blind phase III trial. J Clin Oncol 30:134-141.

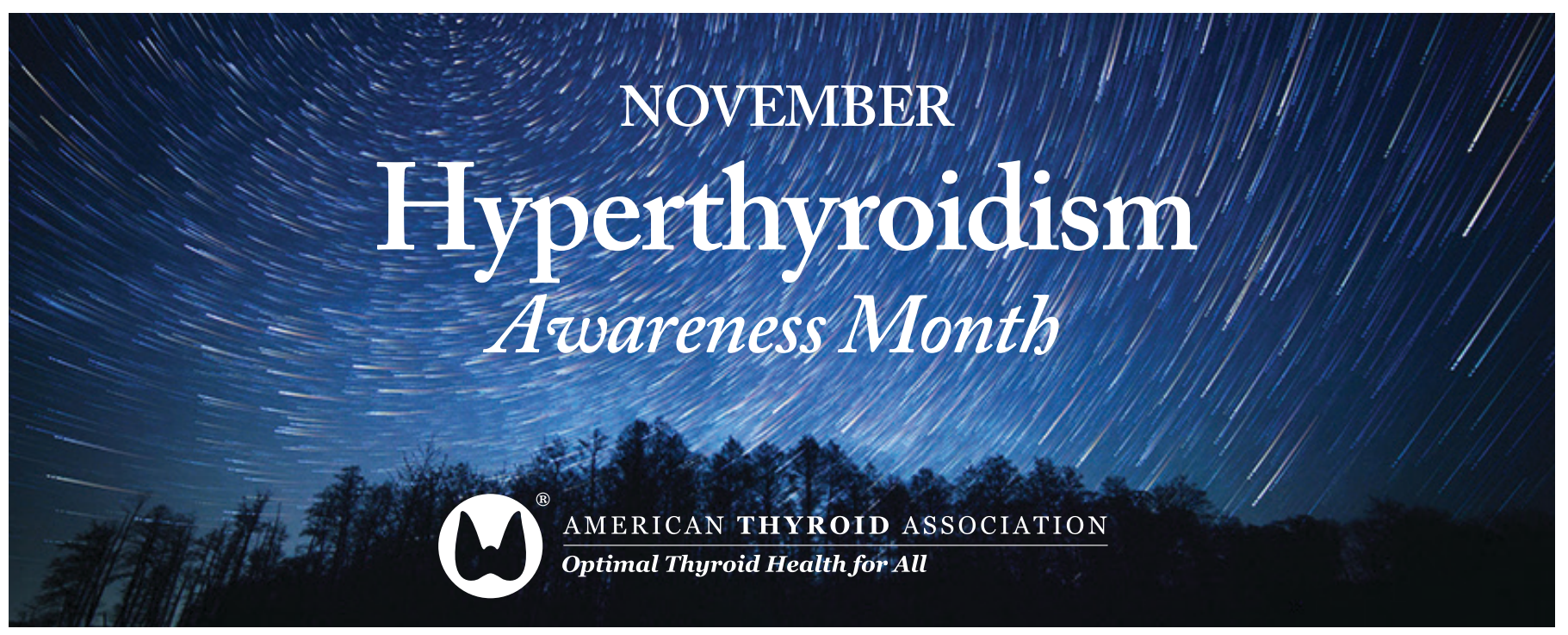

